\title{
The effects of total pleural covering on pneumothorax recurrence and pulmonary function in lymphangioleiomyomatosis patients without history of pleurodesis or thoracic surgeries for pneumothorax
}

\author{
Emily Suzuki $^{1 \wedge}$, Masatoshi Kurihara ${ }^{1,2} \wedge$, Kenji Tsuboshima ${ }^{1,2} \wedge$, Kenichi Watanabe ${ }^{1,2}$, \\ Shouichi Okamoto ${ }^{1,2,3}$, Kuniaki Seyama ${ }^{2,3}$ \\ ${ }^{1}$ Pneumothorax Research Center and Division of Thoracic Surgery, Nissan Tamagawa Hospital, Tokyo, Japan; ${ }^{2}$ Study Group for Pneumothorax and \\ Cystic Lung Diseases, Tokyo, Japan; ${ }^{3}$ Division of Respiratory Medicine, Juntendo University Faculty of Medicine and Graduate School of Medicine, \\ Tokyo, Japan \\ Contributions: (I) Conception and design: E Suzuki, M Kurihara, K Seyama; (II) Administrative support: M Kurihara; (III) Provision of study materials \\ or patients: M Kurihara, K Tsuboshima, K Watanabe, K Seyama; (IV) Collection and assembly of data: E Suzuki, M Kurihara, K Tsuboshima, K \\ Watanabe, S Okamoto, K Seyama; (V) Data analysis and interpretation: E Suzuki, K Tsuboshima, K Seyama; (VI) Manuscript writing: All authors; (VII) \\ Final approval of manuscript: All authors. \\ Correspondence to: Masatoshi Kurihara, MD, PhD. Pneumothorax Research Center and Division of Thoracic Surgery, Nissan Tamagawa Hospital, \\ 4-8-1 Seta Setagaya-ku, Tokyo 158-0095, Japan. Email: kuri@tf6.so-net.ne.jp.
}

Background: Total pleural covering (TPC) is an innovative surgical procedure in which the entire
visceral pleura is wrapped with sheets of oxidized regenerated cellulose (ORC) mesh under video-assisted
thoracoscopic surgery. We have previously reported that TPC could successfully prevent pneumothorax
recurrence in patients with lymphangioleiomyomatosis (LAM). However, the actual efficacy and preventive
effect of TPC on pneumothorax recurrence remains unclear as many LAM patients already had pleural
adhesion prior to TPC that was induced by thoracic surgery and/or pleurodesis. The purpose of this study is
to evaluate the effects of TPC on pneumothorax recurrence and pulmonary function in LAM patients with
no history of thoracic surgeries or pleurodesis.

Methods: We retrospectively reviewed medical charts of 52 patients (60 hemithoraces) who underwent TPC at our center, from January 2003 to September 2019, as a first surgical intervention for pneumothorax.

Results: Pneumothorax recurrence occurred in 12 patients [14 of 60 hemithoraces (23.3\%)] during the observation period $[27$ months $(14.7 ; 56.4)=$ median (lower; upper quartiles)]. The probability of recurrence-free hemithorax post TPC was $81.1 \%$ at 2.5 years and $64.1 \%$ at 5 years. TPC did not produce a significant decrease in either VC \% predicted (pred) or $\mathrm{FEV}_{1} / \mathrm{FVC}$. The pre- $v$ s. post-TPC median (lower; upper quartiles) VC \%pred was $85.7 \%$ (79.7; 98.0) vs. 87.2\% (72.3; 95.6), $\mathrm{P}=0.535$ and the $\mathrm{FEV}_{1} / \mathrm{FVC}$ was 84.6\% (75.7; 89.6) vs. 83.0\% (71.8; 87.0), $\mathrm{P}=0.667$. Mechanistic/mammalian target of rapamycin (mTOR) inhibitors (mTORI) were subsequently initiated in 19 patients $(36.5 \%)$ because of the progression of LAM. The postoperative $\mathrm{FEV}_{1}$ \% pred was significantly lower in patients who required mTORI than in those who did not $[68.1 \%(57.3 ; 82.9)$ vs. 88.7\% (84.6; 89.8), $\mathrm{P}=0.020]$; the decline rate in $\mathrm{FEV}_{1}$ \% pred/year from pre to post TPC was significantly greater in LAM patients who required mTORI than in those who did not [-9.37\% (-4.73; 12.9) vs. $-1.94 \%(1.52 ;-4.50), \mathrm{P}=0.029]$. Postoperative complications were found in 25 of 52 hemithoraces $(48.1 \%)$.

\footnotetext{
$\wedge$ ORCID: Emily Suzuki, 0000-0003-3987-4699; Masatoshi Kurihara, 0000-0001-7192-4743; Kenji Tsuboshima, 0000-0003-0527-4265; Shouichi Okamoto, 0000-0001-8721-0382.
} 


\begin{abstract}
Conclusions: TPC can prevent pneumothorax recurrence without causing ventilatory impairment or severe pleural symphysis in LAM patients. TPC is an effective treatment option for LAM-associated pneumothorax based on its efficacy and safety.
\end{abstract}

Keywords: Total pleural covering (TPC); pulmonary function; lymphangioleiomyomatosis (LAM); pneumothorax; mTOR inhibitor

Submitted Jun 23, 2020. Accepted for publication Nov 04, 2020.

doi: $10.21037 /$ jtd-20-2286

View this article at: http://dx.doi.org/10.21037/jtd-20-2286

\section{Introduction}

Lymphangioleiomyomatosis (LAM) is a cystic lung disease that predominantly affects middle-aged women (1-3). Pneumothorax and progressive lung function impairment are major complications in LAM. Considering the high recurrence rate of pneumothorax, a guideline on LAM diagnosis and management by the American Thoracic Society (ATS) and Japanese Respiratory Society (JRS) recommends ipsilateral pleurodesis following an initial pneumothorax rather than waiting for a recurrent pneumothorax $(1,4)$. However, patients who had undergone lung transplantation following chemical or surgical pleurodesis experienced pleural-related postoperative complications, which indicates extensive pleural adhesion is an increased risk in lung transplantation (5). As an alternative treatment to pleurodesis, total pleural covering (TPC) was invented to prevent pneumothorax recurrence without causing pleural adhesion in LAM patients (6). TPC is a surgical technique in which the entire visceral surface of the lung is wrapped with sheets of oxidized regenerated cellulose (ORC) mesh (Ethicon SURGICEL ${ }^{\circledR}$ absorbable Hemostat gauze, Johnson \& Johnson, Brunswick, NJ, USA) under video-assisted thoracoscopic surgery (VATS) (6). Kim et al. (7) recently reported that lung transplantation after bilateral TPC on a secondary pneumothorax in tuberous sclerosis complex (TSC)-associated LAM resulted in no postoperative complications.

In our previous study, we evaluated the effects of TPC on preventing pneumothorax recurrence and pulmonary function (6). However, the actual efficacy of TPC remains unclear since many LAM patients had thoracic surgeries and/or pleurodesis performed prior to TPC, which causes pleural adhesion and hampers the efficacy of TPC. Therefore, the purpose of this study is to evaluate the effects of TPC on pneumothorax recurrence and pulmonary function in LAM patients with no history of thoracic surgeries or pleurodesis.

We present the following article in accordance with the STROBE reporting checklist (available at http://dx.doi. org/10.21037/jtd-20-2286).

\section{Methods}

\section{Study design and sample}

Between January 2003 and September 2019, 74 patients (95 hemithoraces) pathologically diagnosed with LAM underwent TPC at the Pneumothorax Research Center, Nissan Tamagawa Hospital, Japan. The medical charts of all these patients were reviewed to determine the subset that had not undergone prior interventions that could impede the efficacy of TPC in terms of pneumothorax recurrence and pulmonary function. Figure 1 illustrates the study eligibility criteria as well as the analytical approach that was used to evaluate the true efficacy of TPC on pneumothorax recurrence and pulmonary function in LAM patients. Patients and hemithoraces that had chemical or surgical pleurodesis and/or any surgical interventions prior to TPC were excluded. Pneumothorax recurrence was counted only when patients had been diagnosed via chest X-ray or computed tomography.

\section{$T P C$}

TPC is a surgical technique in which the entire visceral pleura of the lung is wrapped with sheets of ORC mesh under VATS, thereby reinforcing the affected visceral pleura and preventing pneumothorax recurrence. TPC was invented to prevent pneumothorax recurrence without causing frank pleural adhesion in LAM patients and its technical procedure has already been described in detail in our previous study (6). Over 10 sheets of 3" 4 " ORC mesh are required to perfectly wrap the whole visceral pleura in 


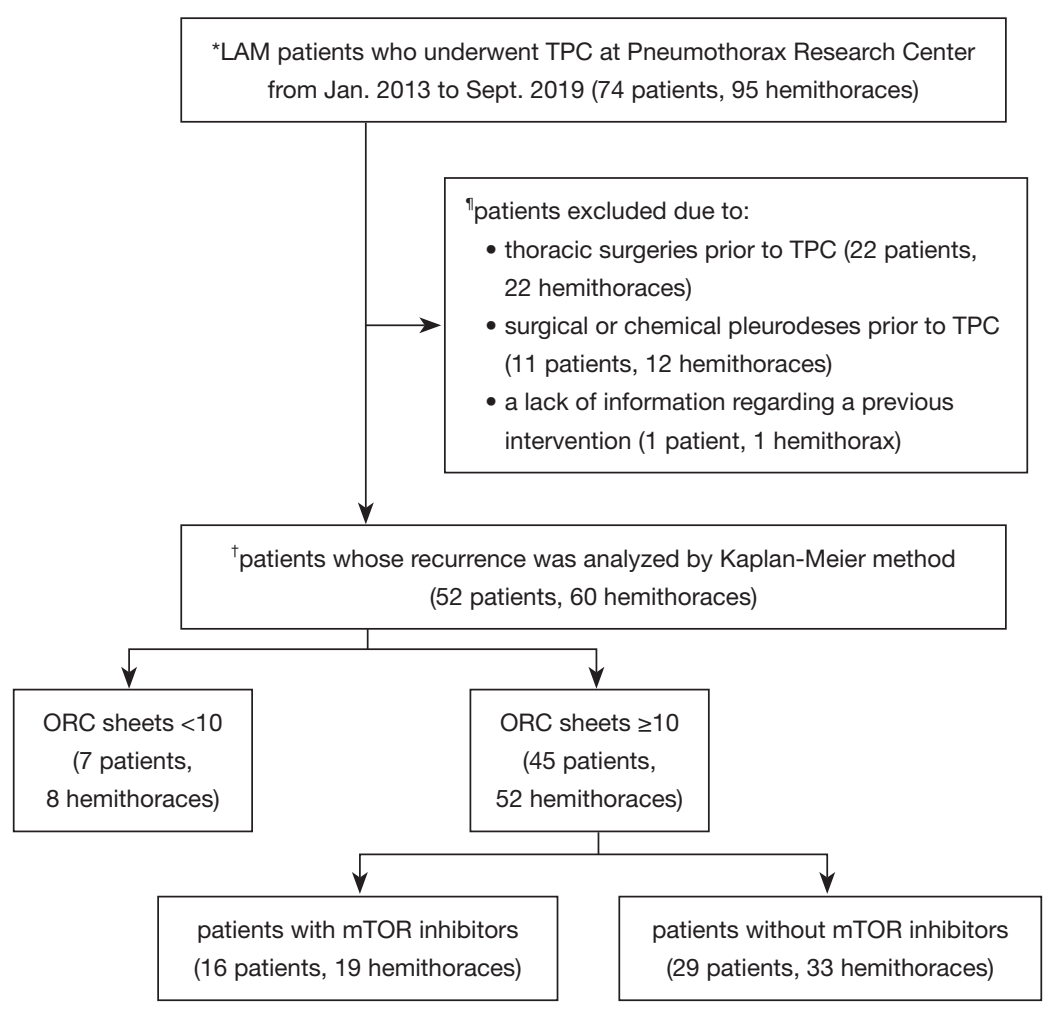

Figure 1 Study eligibility criteria and flow of data analysis. * II Note that the number of LAM patients whose recurrence was estimated by the Kaplan-Meier method $(n=52)^{\dagger}$ is not (the number of LAM patients at the initial evaluation)* minus (the number of LAM patients excluded due the reasons listed) ${ }^{\mathbb{I}}$. This occurred because LAM patients who had TPC in both hemithoraces were either included or excluded, depending on the absence or existence of prior interventions. LAM, lymphangioleiomyomatosis; TPC, total pleural covering; ORC, oxidized regenerated cellulose; mTOR, mechanistic/mammalian target of rapamycin.

a hemithorax. The number of sheets of ORC mesh applied for TPC appeared to influence the subsequent probability of pneumothorax recurrence; TPC with $<10$ sheets of ORC mesh resulted in a higher probability of pneumothorax recurrence than TPC performed with $\geq 10$ sheets (6). An insufficient number of ORC mesh sheets had been used for TPC in some cases due to insufficient lung lobulation and/or pleural adhesion. Postoperative complication was identified using the Clavien-Dindo classification system (8).

\section{Pulmonary function tests (PFT)}

Vital capacity (VC), forced expiratory volume in 1 second $\left(\mathrm{FEV}_{1}\right)$, and forced vital capacity (FVC) were measured by spirometry (CHESTAC-8900; Chest M.I. Inc., Tokyo, Japan). Diffusing capacity for carbon monoxide (DLco) was assessed using a single breath method. The reference values obtained from the Japanese Respiratory Society (9) were utilized to calculate the $\%$ predicted (pred) values.

\section{Statistical analysis}

The Mann-Whitney U-test was used to compare continuous variables (i.e., age and pulmonary function). They were expressed as median (lower; upper quartiles) where appropriate. The probability of pneumothorax recurrence-free hemithorax post TPC was estimated by the Kaplan-Meier method. Log-rank tests were conducted to determine the probability of recurrence-free hemithorax post TPC, both overall and between groups based on the number of ORC sheets used in TPC $(\geq 10$ ORC $v s$. $<10$ ORC). A P value $<0.05$ was considered statistically significant. All statistical analyses were performed using the software 'EZR,' which is a graphical user interface for R (The R foundation for Statistical Computing) (10).

\section{Ethical statement}

This study was approved by the ethics committee of Nissan 
Table 1 Characteristics of study participants

\begin{tabular}{|c|c|c|c|}
\hline Variable & Full sample (N=52 pts/60 hts) & ORC $\geq 10$ group ( $n=45 \mathrm{pts} / 52 \mathrm{hts}$ ) & ORC $<10$ group ( $n=7 \mathrm{pts} / 8 \mathrm{hts})$ \\
\hline Age at $1^{\text {st }}$ PTX, yr & $31.5(28.0 ; 36.0)^{\dagger}$ & $32.0(28.0 ; 36.0)^{\dagger}$ & $30.5(28.8 ; 34.0)^{\dagger}$ \\
\hline Age at TPC, yr & $33.5(30.8 ; 37.0)^{\dagger}$ & $34.0(30.8 ; 37.3)^{\dagger}$ & $32.0(30.8 ; 34.0)^{\dagger}$ \\
\hline \multicolumn{4}{|l|}{ Type of LAM } \\
\hline Side of TPC (R/L) & $31 / 29$ & $25 / 27$ & $6 / 2$ \\
\hline \multicolumn{4}{|l|}{ Clinical features after TPC } \\
\hline \multicolumn{4}{|l|}{ Comorbidities, \# pts } \\
\hline Chylothrax ${ }^{\ddagger}$ & 4 & 4 & 0 \\
\hline Present and past smoker, \# pts & 7 & 6 & 1 \\
\hline Recurrence of PTX & $12 \mathrm{pts} / 14 \mathrm{hts}$ & $8 \mathrm{pts} / 9$ hts & $4 \mathrm{pt} / 5 \mathrm{hts}$ \\
\hline Sporadic/TSC, \# pts & $12 / 0$ & $8 / 0$ & $4 / 0$ \\
\hline $\begin{array}{l}\text { Period from TPC to PTX recurrence, } \\
\text { mths }\end{array}$ & $21(7 ; 46.3)^{\dagger}$ & $21(9.5 ; 50)^{\dagger}$ & $14(4.5 ; 44.5)^{\dagger}$ \\
\hline mTORI initiated ${ }^{\S}$ & $19 \mathrm{pts} / 23 \mathrm{hts}$ & $16 \mathrm{pts} / 19 \mathrm{hts}$ & $3 \mathrm{pts} / 4 \mathrm{hts}$ \\
\hline $\begin{array}{l}\text { Period from TPC to mTORI initiation, } \\
\text { mths }\end{array}$ & $45(17 ; 103)$ & $29(13 ; 67)$ & $126(60.8 ; 144)^{\dagger}$ \\
\hline
\end{tabular}

Tamagawa Hospital, Tokyo, Japan (No. 2019-015) and patient consent was waived. The study was conducted in accordance with the Declaration of Helsinki (as revised in 2013).

\section{Results}

\section{Patient characteristics}

Seventy-four LAM patients (95 hemithoraces) underwent TPC at our center from January 2003 to September 2019. All of them were women, aged 21 to 59 at time of TPC. Twenty-two patients (35 hemithoraces) were excluded from the analyses due to various interventions prior to TPC: bullectomy (20 hemithoraces); TPC at another hospital (2 hemithoraces); surgical pleurodesis (4 hemithoraces); chemical pleurodesis (8 hemithoraces); and an intervention with no detailed information available in the medical chart ( 1 hemothorax). The remaining 52 eligible patients (60 hemithoraces) were retrospectively evaluated in this study (Figure 1). Characteristics of the study sample are shown in Table 1. Forty-six patients had been diagnosed with sporadic LAM whereas 6 patients had TSC-associated LAM. The median (lower; upper quartiles) age at TPC was $33.5(30.8 ; 37.0)$ years. Since our previous study demonstrated that the probability of pneumothorax recurrence post TPC was lower among hemithoraces when 10 or more sheets of 3 " $\times 4$ " ORC were applied for TPC (6), the 52 patients (60 hemithoraces) were categorized into 2 groups based on the number of ORC sheets used for TPC: patients with $<10$ ORC sheets ( $\mathrm{O}=7,8$ hemithoraces) and patients with $\geq 10$ ORC sheets ( $\mathrm{O}=45,52$ hemithoraces) (Figure 1). Eventually, 29 patients (35 hemithoraces) were overlapped with those in our previous study (6), while 23 patients (25 hemithoraces) were unique to this study. 


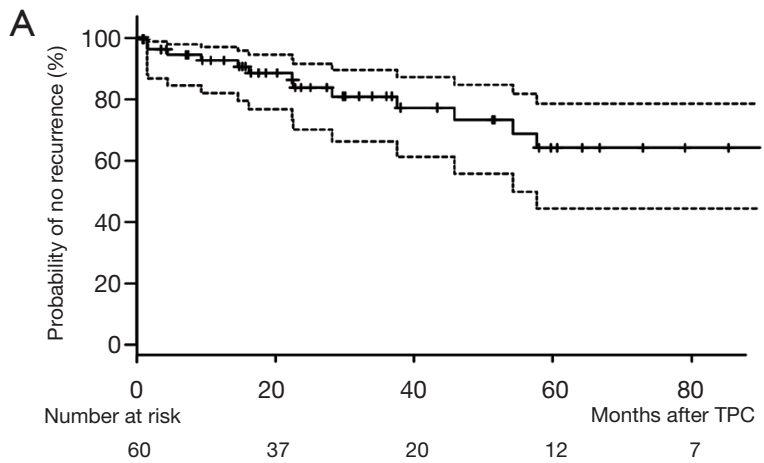

B

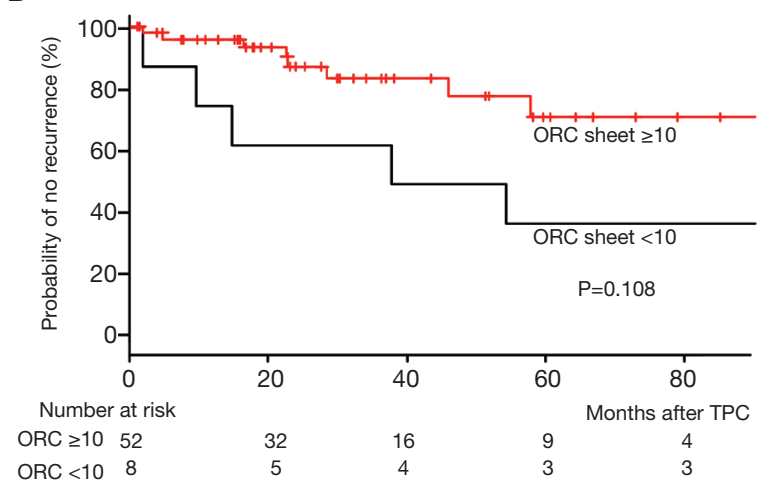

Figure 2 Kaplan-Meier estimate of the probability of recurrencefree hemithorax post TPC. (A) The overall probability of no pneumothorax recurrence among all 60 hemithoraces. Dotted lines show the range of the $95 \%$ confidence interval. (B) The probability of no pneumothorax recurrence, comparing hemithoraces in the ORC $\geq 10$ sheets $v s$. ORC $<10$ sheets groups. The number of subjects being followed at each time interval was provided as number at risk. TPC, total pleural covering; ORC, oxidized regenerated cellulose.

\section{Pneumothorax recurrence post TPC}

We found pneumothorax recurrence in 12/46 (26.1\%) of the sporadic LAM patients but no recurrence in the 6 TSC-associated LAM patients. The overall recurrence rate based on number of hemithoraces was 14/60 hemithoraces (23.3\%) over the median 27-month [14.7; 56.4 (lower; upper quartiles)] observation period (Table 1). The overall probability of recurrence-free hemithorax $(\mathrm{n}=52$ patients, 60 hemithoraces), was $81.1 \%$ at 2.5 years and $64.1 \%$ at 5 years post TPC (Figure $2 A$ ). The probability of recurrence-free hemithorax post TPC in the ORC $\geq 10$ group ( $\mathrm{n}=45$ patients, 52 hemithoraces) was $83.8 \%$ at 2.5 years and $71.7 \%$ at 5 years, while the probabilities in the ORC $<10$ group ( $\mathrm{n}=7$ patients, 8 hemithoraces) were

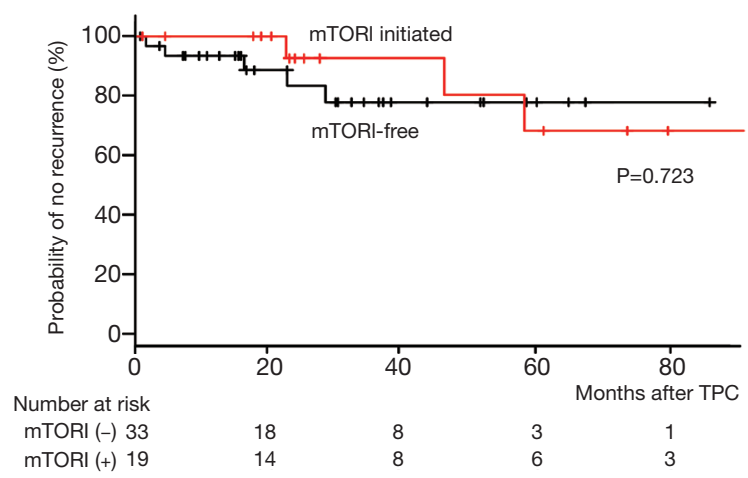

Figure 3 Kaplan-Meier estimate of the probability of recurrencefree hemithorax post TPC in relation to mTORI initiation. Number at risk: the number of subjects being followed at each time interval was provided as number at risk. TPC, total pleural covering; mTORI, mTOR inhibitors.

$62.5 \%$ and $37.5 \%$ respectively at the same time points (Figure 2B). No statistically significant difference in the probability of recurrence-free hemithorax post TPC was found between these two groups $(\mathrm{P}=0.108)$. Compared to our previous report (6) however, the overall probability of recurrence-free hemithorax post TPC was slightly lower at 5 years in the present study, but still resides within the $95 \%$ confidence interval of our previous result (6).

We next calculated the probability of recurrence-free hemithorax post TPC in relation to the potential influence of mTOR inhibitors (mTORI). When we analyzed only the $\geq 10$ ORC sheets group (Figure 1), 16 patients (19 hemithoraces) began mTORI treatment post TPC: sirolimus for 15 patients (18 hemithoraces) and everolimus for 1 TSC-associated LAM patient (1 hemithorax). The median time period from the date of TPC to first mTORI prescription date in the $\geq 10$ ORC sheets group was 29 (13; 67) months (Table 1). Within the $\geq 10$ ORC sheets group, there was no significant difference in the probability of recurrence-free hemithorax post TPC between patients who initiated mTORI ( $\mathrm{n}=16,19$ hemithoraces) and those who did not ( $\mathrm{n}=29,33$ hemithoraces), $\mathrm{P}=0.723$ (Figure 3).

\section{The impact of TPC on the pulmonary function}

We investigated the impact of TPC on the pulmonary function of patients who had undergone this procedure. In order to determine the true impact of TPC on pulmonary function, we collected PFT data only from patients who had TPC with $\geq 10$ 
Table 2 Results of PFTs post TPC in LAM patients with or without prior contralateral interventions

\begin{tabular}{|c|c|c|c|c|}
\hline Variable & Group A (9 pts/9hts) & Group B (24 pts/24 hts) & Total (33 pts/33 hts) & $P$ value \\
\hline Age at TPC, yr & $34.0(30.0 ; 36.0)$ & $34.0(31.3 ; 43.3)$ & 34.0 (30.0; 37.0) & 0.465 \\
\hline VC (L) & $2.50(2.33 ; 2.57)$ & $2.66(2.29 ; 3.09)$ & $2.57(2.31 ; 2.88)$ & 0.342 \\
\hline VC \%pred (\%) & $71.9(65.8 ; 81.8)$ & $80.8(70.1 ; 93.3)$ & $76.8(65.8 ; 85.6)$ & 0.332 \\
\hline FVC \%pred (\%) & $72.5(64.1 ; 79.5)$ & 82.8 (71.9; 93.0) & 79.5 (64.8; 89.8) & 0.111 \\
\hline $\mathrm{FEV}_{1}(\mathrm{~L})$ & $2.06(1.52 ; 2.23)$ & $2.07(1.78 ; 2.43)$ & $2.06(1.75 ; 2.34)$ & 0.585 \\
\hline $\mathrm{FEV}_{1} \%$ pred (\%) & $69.8(50.7 ; 78.3)$ & 79.8 (61.2; 89.3) & $76.2(60.7 ; 88.2)$ & 0.309 \\
\hline $\mathrm{FEV}_{1} / \mathrm{FVC}(\%)$ & $78.9(69.1 ; 83.0)$ & $83.3(72.5 ; 87.6)$ & $82.8(71.5 ; 87.5)$ & 0.462 \\
\hline
\end{tabular}

Data are presented as median (lower; upper quartiles) values. Group A includes patients who had contralateral interventions prior to TPC whereas Group B had none. As all patients did not have PFTs conducted both pre and post TPC, only post-TPC PFT data were analyzed [33pts (33 hts)]. ${ }^{\dagger}$, DLco results were available for a total of 27 patients (27 hemithoraces): 8 patients (8 hemithoraces) in Group A and 19 patients (19 hemithoraces) in Group B. The period from TPC to post-TPC spirometry was 20 (6; 30.5) for Group A and 13.5 (4.25; 23.8 ) months for Group B. PFT, pulmonary function test; TPC, total pleural covering; LAM, lymphangioleiomyomatosis; VC, vital capacity; FVC, forced vital capacity; $\mathrm{FEV}_{1}$, forced expiratory volume in 1 second; DLco, diffusing capacity for carbon monoxide.

ORC sheets (45 patients, 52 hemithoraces). Additionally, we used only PFT results within 3 years after TPC to minimize the influence of underlying disease progression.

\section{Post-TPC pulmonary function with or without prior contralateral interventions}

Table 2 presents PFT results based on the presence or absence of contralateral interventions prior to TPC. Among the 45 patients ( 52 hemithoraces) in the $\geq 10$ ORC sheets group, post-TPC PFT data were available for 33 patients (33 hemithoraces). The 33 patients and hemithoraces were classified into 2 groups based on their history of prior contralateral interventions: Group A included hemithoraces with contralateral interventions prior to TPC and Group $\mathrm{B}$ included hemithoraces without prior contralateral interventions. In Group A, 9 patients (9 hemithoraces) who underwent TPC as a first surgical intervention on the ipsilateral hemithorax, had had various prior interventions on the contralateral side: TPC as a first intervention $(n=5)$; TPC performed after a history of other interventions $(n=2)$; and thoracic surgeries other than TPC $(n=2)$. We compared the results of post-TPC PFTs between Group A and Group B to measure the influence of prior contralateral interventions on pulmonary function.
Group A patients showed lower pulmonary function in all variables than Group B patients, although none of the differences reached statistical significance. The median postoperative VC \% pred and $\mathrm{FEV}_{1} / \mathrm{FVC}$ in Group B were $80.8 \%$ and $83.3 \%$ respectively, indicating that TPC did not cause either restrictive or obstructive ventilatory impairment.

\section{Comparison between pre- and post-TPC pulmonary function and the rate of change per year}

Among the 24 patients (24 hemithoraces) in Group B, 14 patients (14 hemithoraces) had both pre- and post-TPC PFT data available for analysis. We compared pre- to postoperative pulmonary function for these 14 patients (Table 3).

Although both DLco and DLco \%pred were mildly to moderately impaired at baseline due to LAM, TPC did not make a statistically significant decrease in various measures of pulmonary function within the postoperative 3 years.

\section{Comparison by mTORI initiation during the postoperative period}

The 14 patients (14 hemithoraces) who had both pre- and post-TPC PFT data available for analysis were further classified into 2 groups based on mTORI initiation during the 
Table 3 Pre- $v s$. post-TPC comparison of PFT results

\begin{tabular}{|c|c|c|}
\hline Variable & Total (14 pts/14 hts) & $P$ value \\
\hline Age at $1^{\text {st }} \mathrm{PTX}, \mathrm{yr}$ & $30.5(27.3 ; 35.8)$ & \\
\hline Age at TPC, yr & $33.0(29.0 ; 43.8)$ & \\
\hline VC (L) & & 0.566 \\
\hline Pre & $2.81(2.63 ; 3.63)$ & \\
\hline Post & $2.88(2.28 ; 3.36)$ & \\
\hline VC \%pred (\%) & & 0.535 \\
\hline Pre & 85.7 (79.7; 98.0) & \\
\hline Post & $87.2(72.3 ; 95.6)$ & \\
\hline FVC (L) & & 0.603 \\
\hline Pre & $2.78(2.45 ; 3.63)$ & \\
\hline Post & $2.79(2.28 ; 3.24)$ & \\
\hline FVC \%pred (\%) & & 0.701 \\
\hline Pre & $86.9(78.4 ; 106)$ & \\
\hline Post & $87.1(74.3 ; 97.6)$ & \\
\hline $\mathrm{FEV}_{1}(\mathrm{~L})$ & & 0.301 \\
\hline Pre & $2.40(2.01 ; 2.60)$ & \\
\hline Post & $2.17(1.83 ; 2.47)$ & \\
\hline $\mathrm{FEV}_{1} \%$ pred (\%) & & 0.265 \\
\hline Pre & $86.8(77.5 ; 96.0)$ & \\
\hline Post & 86.5 (64.9; 88.9) & \\
\hline $\mathrm{FEV}_{1} / \mathrm{FVC}(\%)$ & & 0.667 \\
\hline Pre & $84.6(75.7 ; 89.6)$ & \\
\hline Post & $83.0(71.8 ; 87.0)$ & \\
\hline $\operatorname{DLco}(\mathrm{mL} / \mathrm{min} / \mathrm{mmHg})^{\dagger}$ & & 0.699 \\
\hline Pre & $14.9(13.0 ; 18.0)$ & \\
\hline Post & $14.3(12.2 ; 16.4)$ & \\
\hline DLco \%pred $(\%)^{\dagger}$ & & 0.748 \\
\hline Pre & $66.6(54.7 ; 78.7)$ & \\
\hline Post & $64.1(53.2 ; 70.8)$ & \\
\hline
\end{tabular}

Data are presented as median (lower; upper quartiles) values. ${ }^{\dagger}$, pre- and post-DLco results were available for 11 patients. The period from spirometry to TPC was $4.0(0 ; 10)$ months whereas the period from TPC to spirometry post TPC was $10(3.5 ; 18.5)$ months. TPC, total pleural covering; PFT, pulmonary function test; LAM, lymphangioleiomyomatosis; VC, vital capacity; FVC, forced vital capacity; $F V_{1}$, forced expiratory volume in 1 second; DLco, diffusing capacity for carbon monoxide. 3-year follow-up period post TPC (Table 4). Group C included 6 patients (6 hemithoraces) who had mTORI initiated $v s$. Group D with 8 patients (8 hemithoraces) who did not. We compared the PFT results pre and post TPC between these 2 groups. In Group C patients, the postoperative PFT results closest to mTORI initiation were analyzed.

Group C patients tended to have lower preoperative DLco \% pred values than Group D patients $(\mathrm{P}=0.082)$. This suggests that patients in Group $\mathrm{C}$ were in a more advanced stage of LAM than Group D patients prior to TPC. In addition, postoperative $\mathrm{FEV}_{1} / \mathrm{FVC}$ tended to be lower in Group C than in Group D, $\mathrm{P}=0.059$. Notably, postoperative $\mathrm{FEV}_{1} \%$ pred $(\mathrm{P}=0.020)$ as well as the rate of change per year in $\mathrm{FEV}_{1}$ \% pred $(\mathrm{P}=0.029)$ were significantly lower in Group C than in Group D (Table 4).

\section{Pulmonary function after bilateral TPC}

Seven patients underwent bilateral TPCs at different times as the first surgical treatment for pneumothorax on each hemithorax. PFTs were performed after the $2^{\text {nd }}$ TPC in 5 of 7 patients and the median period from the $2^{\text {nd }}$ TPC to spirometry was $9(2 ; 18)$ months. As shown in Table 5 , moderate degrees of restrictive ventilatory impairment and decreased diffusing capacity were present.

\section{Postoperative complications}

Postoperative complications occurred in 25 of 52 hemithoraces (48.1\%); Grade II, 14 hemithoraces (26.9\%), Grade IIIa, 10 hemithoraces (19.2\%), and Grade IIIb, a hemithorax $(0.2 \%)$. The most frequent complications were prolonged postoperative air leak $(n=13)$ defined as persistent air leak beyond 7 days, under Grade II, accounted for $52.0 \%$ of all complications. Grade IIIb complication occurred in the patient with dense pleural adhesion at the time of TPC, requiring reoperation at postoperative day 2 to control bleeding.

\section{Discussion}

This retrospective study focused on the effects of TPC in relation to the prevention of pneumothorax recurrence and postoperative lung function in LAM patients with no history of thoracic surgeries and/or pleurodesis. Unexpectedly, regardless of the number of ORC sheets applied for TPC, 
Table 4 Comparison of PFTs between LAM patients with or without mTORI treatment

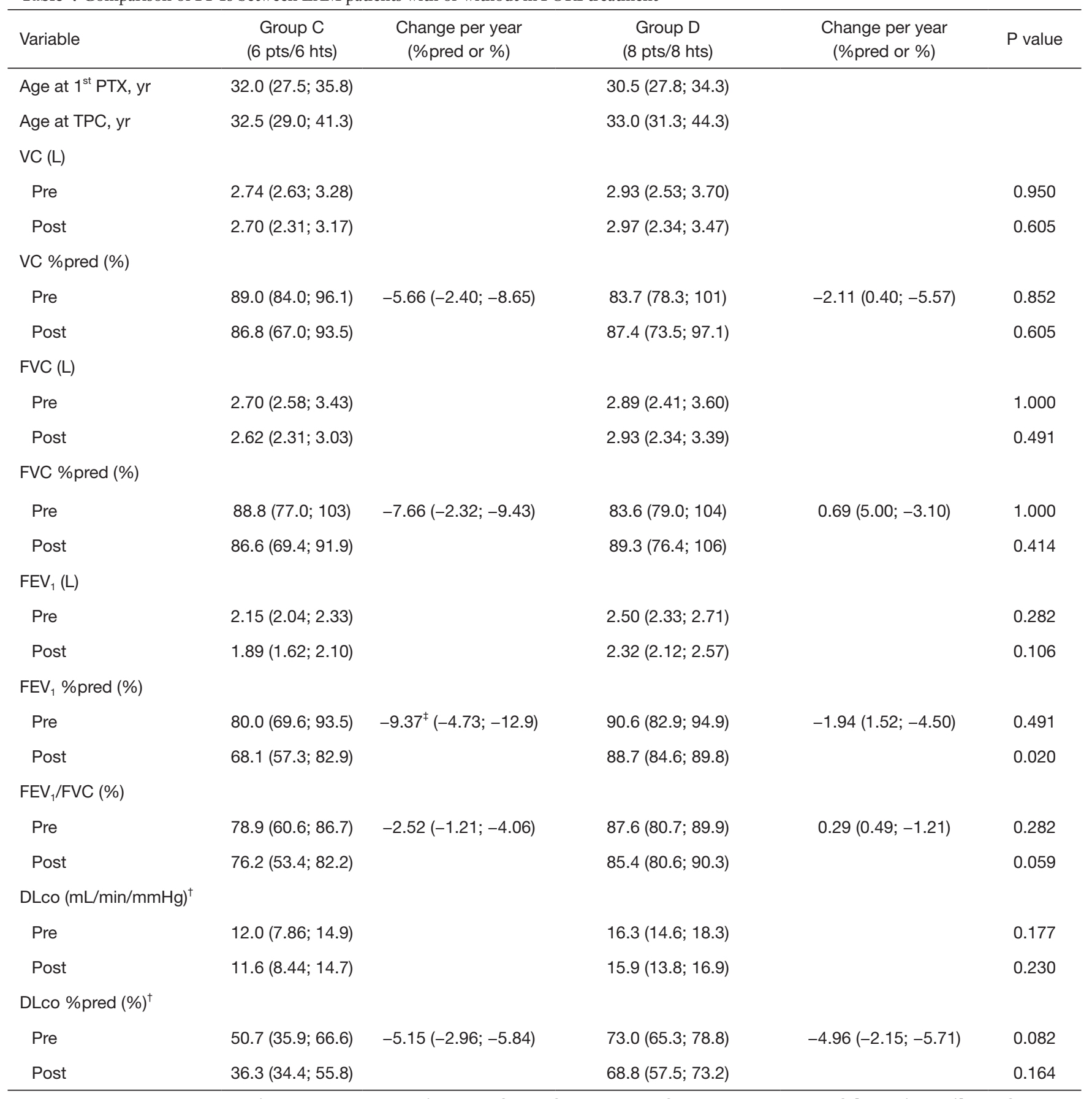

Data are presented as median (lower; upper quartiles) values. Group C received mTORI treatment post-TPC [n=10 (10 hts)] and Group D did not $[\mathrm{n}=14(14 \mathrm{hts})]$. Both pre- and post-TPC PFT data were available for 6 pts $(6 \mathrm{hts})$ in Group C and 8 pts (8 hts) in Group D. ${ }^{\dagger}$, DLco results were available for 3 patients in Group C and 6 patients in Group D; ${ }^{\ddagger}$, change in FEV1 \%pred (\%) per year was significantly greater in Group C than in Group D (P=0.029). The period of months from spirometry to TPC was $4.5(1.25 ; 10)$ and $1(0 ; 5)$ for Groups $C$ and $D$, respectively, and from TPC to spirometry was $13(5.25 ; 27.5)$ and $17(8.75 ; 20.8)$ for Groups $C$ and $D$, respectively. PFT, pulmonary function test; mTORI, mTOR inhibitors; LAM, lymphangioleiomyomatosis; TPC, total pleural covering; VC, vital capacity; FVC, forced vital capacity; $\mathrm{FEV}_{1}$, forced expiratory volume in 1 second; DLco, diffusing capacity for carbon monoxide. 
Table 5 PFT results post bilateral TPC

\begin{tabular}{lc}
\hline Pulmonary function & After bilateral TPC $(\mathrm{n}=5)$ \\
\hline VC (L) & $2.34(2.33 ; 2.57)$ \\
VC \%pred (\%) & $69.3(64.7 ; 75.5)$ \\
FVC (L) & $2.23(2.20 ; 2.38)$ \\
FVC \%pred (\%) & $64.7(64.1 ; 72.5)$ \\
FEV $_{1}(\mathrm{~L})$ & $2.06(1.52 ; 2.21)$ \\
$\mathrm{FEV}_{1} \%$ pred (\%) & $69.8(50.7 ; 78.1)$ \\
$\mathrm{FEV}_{1} / \mathrm{FVC}(\%)$ & $76.3(69.1 ; 82.2)$ \\
$\mathrm{DLCO}(\mathrm{mL} / \mathrm{min} / \mathrm{mmHg})^{\dagger}$ & $12.9(9.9 ; 15.6)$ \\
DLco \%pred $(\%)^{\dagger}$ & $54.2(41.9 ; 65.2)$ \\
\hline
\end{tabular}

Data are presented as median (lower; upper quartiles) values. Post-bilateral TPC PFT data were available for analysis in 5 LAM patients (5 hemithoraces). ${ }^{\dagger}$, DLco results were available for 4 patients. PFT, pulmonary function test; TPC, total pleural covering; LAM, lymphangioleiomyomatosis; VC, vital capacity; FVC, forced vital capacity; $F V_{1}$, forced expiratory volume in 1 second; DLco, diffusing capacity for carbon monoxide.

the probability of recurrence-free hemithorax was slightly lower than the results of our previous analysis, which included TPC outcomes in LAM patients with focal or scattered pleural symphysis (6). One of the possible reasons for this result might be that thoracic surgeons changed during the research period. Six thoracic surgeons conducted TPC under the supervision of the inventor (MK) of TPC. All of them were experienced thoracic surgeons, but 5 had few experiences with thoracic surgery of LAM lungs and 4 had almost no experience performing TPC before joining our hospital. Actually, there are a couple of important checkpoints at which surgeons' skills would influence the total number of ORC sheets used for TPC and the finish of TPC, i.e., whether the entire surface of the lung is fully enclosed by ORC sheets. First, surgeons need to decide the degrees of crinkle of each ORC sheet as well as of the overlap between the adjoining two ORC sheets when applied for the mildly deflated lung surface; the surgeons have to image the lung surface remains entirely wrapped by ORC sheets even after the lung fully expand. Second, they need to decide which orientation of ORC sheet is appropriate to affix to the lung surface place by place. Third, how much fibrin glue should be dropped after the entire lung surface is wrapped. It is true that to assess all these checkpoints is especially challenging to surgeons in training. Another reason may be the retrospective design of our studies that might have produced variability across the 2 studies.

The probability of recurrence-free hemithorax post TPC regardless of the number of ORC sheets used was almost identical in both studies at 2.5 years $(80.8 \%$ in our previous study vs. $81.1 \%$ in this study), and at 5 years the probability still resided within the $95 \%$ confidence interval of the probability found in our previous study (6). The slightly lower recurrence-free probability in the period $>2.5$ years might reflect the characteristics of this study population, particularly the severity of underlying LAM.

Gonano et al. (11) recently reported the probability of recurrence-free hemithorax after surgical or chemical pleurodesis was $68 \%$ at 5 years, which is slightly higher than that of TPC (64.1\%). But still, we are certain of the advantage and superiority of TPC because TPC is free from severe pleural symphysis even if it developed, in contrast with surgical or chemical pleurodesis. Severe pleural adhesions cause difficulty in dissociating the lung from thoracic wall in the subsequent thoracic surgery and even have a risk of massive hemorrhage at lung transplantation. Furthermore, pleurodesis usually results in patchy pleural adhesions rather than uniform pleural adhesion throughout the entire hemothorax. Once pneumothorax recurs in this condition, patchy and severe pleural adhesions make a subsequent treatment very difficult, and then frequently results in the chronic pneumothorax which may profoundly impair lung function. The risk of chronic pneumothorax is apparently more problematic after pleurodesis than TPC, especially in the cystic lung disease with progressive nature and propensity of recurring pneumothorax, just like LAM.

Compared to both the full sample and the ORC $\geq 10$ sheets group, the pneumothorax recurrence-free probability in LAM patients who had TPC with ORC $<10$ sheets was markedly higher than the result in our previous report (6). This may be because we excluded the influence of thoracic interventions prior to TPC, which usually cause severe pleural adhesions and hamper the procedure and efficacy of TPC. In this study, no statistically significant difference was found in the recurrence-free probability post TPC between the ORC $\geq 10$ sheets and ORC $<10$ sheets groups. This is likely due to the small number of LAM patients in the ORC $<10$ sheets group [7 patients ( 8 hemothoraces) vs. 45 patients (52 hemothoraces) in the ORC $\geq 10$ sheets group]. We examined the reasons for the fewer number of ORC sheets applied on the 7 patients ( 8 hemithoraces) and discovered that pleural adhesions due to a repeated pneumothorax were observed only in 2 hemithoraces whereas the remaining 
6 hemithoraces were free from pleural adhesions. We found that these 6 hemithoraces underwent TPC between the years 2003 and 2007, a time period during which our TPC technique had not yet been established. Accordingly, this result may indicate that postoperative pneumothorax recurrences are not influenced simply by the number of ORC sheets utilized for TPC, if TPC is performed on hemithoraces with no severe pleural adhesions.

TPC is inherently prone to cause a restrictive ventilatory impairment since it will make the visceral pleura 3-9 times thicker (12). In order to understand the true impact of TPC on lung function, we analyzed postoperative lung function only in LAM patients whose ipsilateral hemithoraces had no history of thoracic surgeries and/or pleurodesis. Additionally, we carefully evaluated the influence of contralateral interventions on pulmonary function in the LAM patients who had already received some interventions on a contralateral hemithorax. We then found that unilateral TPC showed a marginal impact on VC \%pred and FVC \%pred whereas bilateral TPC caused a moderate degree of restrictive ventilatory impairment. Since there is a trade-off between the lower frequency of pneumothorax recurrence by reinforcement of visceral pleura and the loss of pulmonary function, the lung function impairment after bilateral TPC could still be acceptable.

TPC is an intervention to the pleura, and thus would not prevent the development of parenchymal cysts. Therefore, mTORI has been prescribed to LAM patients in case they need to stabilize pulmonary function after TPC. The ATS/JRS clinical practice guideline recommends prescribing sirolimus if LAM patients have abnormal lung function defined as FEV1 $<70 \%$ pred or progressively declining FEV1 (13). Because of impaired or declining lung function during the subsequent period post TPC, 18 out of the 52 LAM patients were given sirolimus and 1 TSC-associated LAM patient was given everolimus. Indeed, LAM patients who initiated sirolimus or everolimus (Group C) post TPC tended to have lower preoperative DLco \%pred compared to LAM patients who had been free of mTORI (Group D). Additionally, both FEV1 \%pred and the rate of change per year in FEV1 \%pred became significantly lower in Group C than in Group D. Therefore, LAM patients who required mTORI after TPC are likely to suffer from more progressive LAM.

Although the effect of sirolimus on the recurrence of pneumothorax remains unknown, no significant difference found in the postoperative recurrence-free probability may imply that mTORI prevents pneumothorax recurrence in patients with progressive LAM. Zhou et al. (14) reported a similar observation that $5 \mathrm{LAM}$ patients who had experienced repeat episodes of pneumothorax showed no recurrence after initiation of sirolimus therapy. However, further study is needed to determine if sirolimus prevents pneumothorax recurrence because we have treated 3 LAM patients who developed pneumothorax while taking sirolimus (being on sirolimus for 3, 5, and 7 years, respectively) (unpublished data).

TPC could therefore be an effective alternative to control LAM-associated pneumothorax recurrence. The British Thoracic Society guideline for the management of spontaneous pneumothorax recommends simple aspiration and/or small-bore chest tube drainage, depending on the size of pneumothorax, which are both less invasive and less likely to cause severe pleural adhesions (15). TPC could be a useful treatment option for LAM patients who have undergone simple aspiration and/or small-bore chest tube drainage which failed to cure pneumothorax in terms of lower recurrence rate, low morbidity, and not producing severe pleural symphysis. The current ATS/JRS guideline on LAM diagnosis and management suggests that LAM patients be offered ipsilateral pleurodesis after their initial pneumothorax, placing greater value on reduction in morbidity and the costs associated with a recurrent pneumothorax than on the adverse effects of pleurodesis (4). However, the gradual popularization of TPC and its successful applications for intractable pneumothorax resulting from conditions other than LAM (16-20) as well as the use of mTORI, are expected to change future recommendations for the management of LAM-associated pneumothorax to focus on the efficacy of lowering recurrence, safety, and no pleural symphysis.

Our study had several limitations. First, this was a retrospective study performed in a single hospital. The study sample size is relatively small, but we still believe our findings are noteworthy given the rarity of LAM. Second, not all the patients underwent PFTs both pre and post TPC, and therefore a limited amount of PFT data were available for analysis. Third, the remote effects of thickened visceral pleura on pulmonary function and patients' activity remain unknown. To this end, a longer observation period is necessary, but this would be compromised by the progression of underlying LAM.

\section{Conclusions}

We conclude that TPC effectively reduce pneumothorax recurrence without causing ventilatory impairment or pleural adhesion in LAM patients. Our results suggest that 
TPC is an effective treatment option for LAM-associated pneumothorax in terms of the prevention of pneumothorax recurrence and the impact on pulmonary function.

\section{Acknowledgments}

The authors would like to thank Ms. Elaine Blumberg for her excellent editing and proofreading of English writing. Funding: None.

\section{Footnote}

Reporting Checklist: The authors have completed the STROBE reporting checklist. Available at http://dx.doi. org/10.21037/jtd-20-2286

Data Sharing Statement: Available at http://dx.doi. org/10.21037/jtd-20-2286

Peer Review File: Available at http://dx.doi.org/10.21037/jtd20-2286

Conflicts of Interest: The authors have completed the ICMJE uniform disclosure form (http://dx.doi.org/10.21037/jtd-202286). The authors have no conflicts of interest to declare.

Ethical Statement: The authors are accountable for all aspects of the work in ensuring that questions related to the accuracy or integrity of any part of the work are appropriately investigated and resolved. This study was approved by the ethics committee of Nissan Tamagawa Hospital, Tokyo, Japan (No.2019-015) and patient consent was waived. The study was conducted in accordance with the Declaration of Helsinki (as revised in 2013).

Open Access Statement: This is an Open Access article distributed in accordance with the Creative Commons Attribution-NonCommercial-NoDerivs 4.0 International License (CC BY-NC-ND 4.0), which permits the noncommercial replication and distribution of the article with the strict proviso that no changes or edits are made and the original work is properly cited (including links to both the formal publication through the relevant DOI and the license). See: https://creativecommons.org/licenses/by-nc-nd/4.0/.

\section{References}

1. McCormack FX. Lymphangioleiomyomatosis: a clinical update. Chest 2008;133:507-16.

2. Taveira-DaSilva AM, Hedin C, Stylianou MP, et al. Reversible airflow obstruction, proliferation of abnormal smooth muscle cells, and impairment of gas exchange as predictors of outcome in lymphangioleiomyomatosis. Am J Respir Crit Care Med 2001;164:1072-6.

3. Taveira-DaSilva AM, Stylianou MP, Hedin CJ, et al. Maximal oxygen uptake and severity of disease in lymphangioleiomyomatosis. Am J Respir Crit Care Med 2003;168:1427-31.

4. Gupta N, Finlay GA, Kotloff RM, et al. Lymphangioleiomyomatosis Diagnosis and Management: High-Resolution Chest Computed Tomography, Transbronchial Lung Biopsy, and Pleural Disease Management. An Official American Thoracic Society/ Japanese Respiratory Society Clinical Practice Guideline. Am J Respir Crit Care Med 2017;196:1337-48.

5. Almoosa KF, Ryu JH, Mendez J, et al. Management of pneumothorax in lymphangioleiomyomatosis: effects on recurrence and lung transplantation complications. Chest 2006;129:1274-81.

6. Kurihara M, Mizobuchi T, Kataoka H, et al. A Total Pleural Covering for Lymphangioleiomyomatosis Prevents Pneumothorax Recurrence. PLoS One 2016;11:e0163637.

7. Kim DH, Ahn HY, Son BS, et al. Total pleural coverage followed by lung transplantation in patient with lymphangioleiomyomatosis. Gen Thorac Cardiovasc Surg 2020;68:1208-11.

8. Dindo D, Demartines N, Clavien PA. Classification of surgical complications: a new proposal with evalutation in a cohort of 6336 patients and results of a survey. Ann Surg 2004:240:205-13.

9. Sharp DS. Reference values for lung function in the Japanese: recording of normal lung function from 14 institutes in Japan. Jpn J Thoracic Dis 1993;31:421-7.

10. Kanda Y. Investigation of the freely available easy-touse software 'EZR' for medical statistics. Bone Marrow Transplant 2013;48:452-8.

11. Gonano C, Pasquier J, Daccord C, et al. Air travel and incidence of pneumothorax in lymphangioleiomyomatosis. Orphanet J Rare Dis 2018;13:222.

12. Ebana H, Hayashi T, Mitani K, et al. Oxidized regenerated cellulose induces pleural thickening in patients with pneumothorax: possible involvement of the mesothelialmesenchymal transition. Surg Today 2018;48:462-72.

13. McCormack FX, Inoue Y, Moss J, et al. Efficacy and safety of sirolimus in lymphangioleiomyomatosis. $\mathrm{N}$ Engl J Med 2011;364:1595-606. 
14. Zhou L, Ouyang R, Luo H, et al. Efficacy of sirolimus for the prevention of recurrent pneumothorax in patients with lymphangioleiomyomatosis: a case series. Orphanet J Rare Dis 2018; $13: 168$.

15. MacDuff A, Arnold A, Harvey J, et al. Management of spontaneous pneumothorax: British Thoracic Society Pleural Disease Guideline 2010. Thorax 2010;65 Suppl 2:ii18-31.

16. Mizobuchi T, Kurihara M, Ebana H, et al. A total pleural covering of absorbable cellulose mesh prevents pneumothorax recurrence in patients with Birt-HoggDube syndrome. Orphanet J Rare Dis 2018;13:78.

17. Kadota Y, Fukui E, Kitahara N, et al. Total pleural covering technique for intractable pneumothorax in patient with Ehlers-Danlos syndrome. Gen Thorac Cardiovasc

Cite this article as: Suzuki E, Kurihara M, Tsuboshima K, Watanabe K, Okamoto S, Seyama K. The effects of total pleural covering on pneumothorax recurrence and pulmonary function in lymphangioleiomyomatosis patients without history of pleurodesis or thoracic surgeries for pneumothorax. J Thorac Dis 2021;13(1):113-124. doi: 10.21037/jtd-20-2286
Surg 2016;64:425-8.

18. Noda M, Okada Y, Maeda S, et al. A total pleural covering technique in patients with intractable bilateral secondary spontaneous pneumothorax: Report of five cases. Surg Today 2011;41:1414-7.

19. Kusu T, Nakagiri T, Minami M, et al. Null allele alpha-1 antitrypsin deficiency: case report of the total pleural covering technique for disease-associated pneumothorax. Gen Thorac Cardiovasc Surg 2012;60:452-5.

20. Takegahara K, Yoshino N, Usuda J. A Case of Recurrent Pneumothorax Associated with Birt-Hogg-Dube Syndrome Treated with Bilateral Simultaneous Surgery and Total Pleural Covering. Ann Thorac Cardiovasc Surg 2017;23:309-12. 